\title{
Building Electronic Educational Environments: Why, What and How?
}

\author{
Tom J. van Weert and Bauke van der Wal \\ Expert Centre for Educational Technology, Innovation and Training; Hogeschool van Utrecht, \\ University for Professional Education and Applied Science
}

Key words: Electronic Learning Environment, Learning Organisation, LearningSpace, New Media, Organisation of Learning, Requirements

\begin{abstract}
The workplace is becoming a learning organisation empowered by the use of information technology. And education is becoming "the" learning organisation, sharing many characteristics with this new workplace. Added value in new forms of education lies in more interactivity and feedback loops, in reorientation of the "process" of learning and in the use of meta-structures. New computer-based media contribute to this added value by providing dynamic simulation environments or virtual worlds.
\end{abstract}

Higher education seems to be aware of the need of educational innovations supported by Information and Communication Technology (ICT). The question is whether it is capable of changing itself into "the" new learning organisation, a student-centred working environment in which ICT is integrated. ICT support should provide functionality appropriate for this new learning situation. Requirements for electronic learning environments, such as LearningSpace, are presented and a comparison between environments is made.

\subsection{Introduction}

In education, just as in business and industry, Information and Communication Technology (ICT) is often used because it is there. In such cases, we may see lecture rooms with computer screens for the students and

The original version of this chapter was revised: The copyright line was incorrect. This has been corrected. The Erratum to this chapter is available at DOI: 10.1007/978-0-387-35502-3_19 
a master computer, controlling these screens, for the lecturer; the computer is automating the lecture hall.

However, experience in business and industry has shown that the real strength of information technology lies not in automating existing work processes, but in empowering people to change those work processes. Here is a lesson to be learned by education.

Experience also shows that information technology cannot be separated from its application, that it cannot be separated from the processes it is used for nor from its organisational context. Its functionality should fit the people, the processes, and the organisation. The question then is "What are effective electronic educational environments?" What are the characteristics of such environments that will fit students as well as teachers, and that will fit study process as well as educational organisation?

\subsection{The workplace: a learning organisation}

Our society has developed from one of relative stability, in which wellknown problems were solved over and over again (industrial society), to one of change. New problems that need to be solved are appearing each day (information society). This change has had an effect on organisational structures. In order to be able to cope with change, lean, team-based organisations have appeared with participating management. The traditional hierarchical organisation, with its fixed structure reflecting well-known knowledge, is changing into a flat, team-based organisation. Such organisations operate as knowledge networks in which new knowledge is perpetually assimilated; these organisations are "learning organisations."

Work in these new organisations has changed and workers are in need of new competencies [1, pages 83-101]. Basically, they need the competency of Lifelong Learning (LLL): working and learning turn into one. In such new work environments, actions and results cannot be assessed in the conventional way against time and standard examples. Instead, workers must account for their actions and results against agreed criteria of quality, specific for the task at hand. They assess themselves against these criteria. In turn, their assessment is reviewed by peers and experts in the light of agreed criteria.

Work in this new type of learning organisation is empowered by the use of information technology. This technology supplies an electronic work environment in which communication tools, personal tools, and professionspecific tools greatly enhance individual and group effectiveness in the application of knowledge and the assessment of results. 


\subsection{Education: "the" learning organisation}

Societal developments are also affecting education, in both content and organisation [2]. Educational organisation is changing from a hierarchybased, teacher-centred type of organisation towards a student-centred, network type of organisation. And for good reason: learning by heart of static knowledge, delivered by the teacher, to be applied only later in your job environment, is no longer effective. Instead, you must learn to apply knowledge directly in all kinds of problem situations. We are moving from knowledge as a static entity to dynamic knowledge, i.e., the application of knowledge. Learning and working environments are growing together: working is applying new knowledge, and learning is also applying new knowledge. The working environment is a learning organisation, but education is "the" learning organisation.

"The" new learning organisation shares many characteristics with the new working place. Coached by teachers, students learn to apply knowledge in a setting for solving realistic problems. They learn to apply knowledge in teams, and are part of a communication network in which peers and experts share knowledge and experience. When moving to a job before or after graduation, students find themselves again in learning organisations; their learning is lifelong.

Content (knowledge) shifts to process (application of knowledge). The focus of the learning process shifts from specific content, which may vary with the problem at hand, to the organisation of the learning. This implies that students need organisers to structure their learning process. Also their specific, individual achievements will be filed into portfolios. They will communicate with peers and experts, both at university and at home. They will use professional tools to solve realistic problems, thus learning to apply new knowledge. And teachers who are working in "the" learning organisation will have to change their focus from delivering content to coaching the learning process.

\subsection{Value-added education}

Some will argue that the vision of education that we present here is extreme. Why is there a need to change? After all, in traditional education students and teachers may benefit from information technology through "automation" of the existing educational process.

But how promising is this line of reasoning? Let us make a comparison with the publishing business. Many publishers still publish in the same way as Gutenberg did, but with the addition of digital production and delivery mechanisms. Afraid to be left out of future markets, publishers use these 
mechanisms to produce more and more of the same. According to Berghel [3], they believe in a twisted variation of Metcalfe's Law (the value of the Internet increases with the square of the number of nodes): the value of electronic publishing increases with the square of the number of documents on the Web. Although some publishers claim benefits from electronic publishing, such as lower overhead and production costs, a wider variety of advertising and marketing (broadcasting, narrowcasting, "personal-casting"), Berghel judges the value of electronic publishing to vary inversely with the number of Web nodes.

A lesson is to be learned here: automation of the original process does not necessarily bring success. Real success lies in added value. In the case of electronic publishing, Berghel sees this added value in:

- addition of interactivity and feedback loops;

- a reorientation of provider and consumer on the "process" of publishing rather than on individual products and services;

- meta-structures of syntax and semantics used in this publishing process. Early applications of value-added publishing are:

- publications with limited commercial appeal;

- publications with narrow audience appeal;

- personal digital digest ("digital cuttings" to your personal taste);

- personalised retrieval publications (such as a personalised encyclopaedia);

- home grown, personal publications;

- interactive publications.

This implies that added value is to be found in the publishing process, and not so much in the published content.

An analogy can be made with education. Who needs yet another university on the Internet offering specific course content that does not fit your requirements and your culture? Added value in education is to be found in:

- addition of interactivity and feedback loops;

- a reorientation of teacher and student on the "process" of learning rather than on individual products and services;

- use of meta-structures of syntax and semantics in the learning process.

\subsection{The role of new media in education: what I see is what I simulate}

The focus in value-added education is on the learning process, on interactivity and feedback loops. Here new media can make an important contribution. The new, computer-based media allow realisation of dynamic simulation environments or virtual worlds: "WISIWIS, What I See Is What 
I Simulate". The strength of the new media is that they allow us to "virtualise" problem specifications or solution statements in a flexible, nonphysical way. A great advantage of this virtualisation, realised, for example with add-ons or plug-ins on the World Wide Web, is reusability and consistency of simulation. Simulation of processes is supported by, for example, Java-applets, ("shocked") Macromedia applications or additional programs, and may be delivered to the students on CD-ROM. In a larger perspective, simulation is also possible through complete environments. An example is "the Cave" at Virginia Tech [4], a simulation environment for research on the mechanical behaviour of materials.

A. P. Ershov was the first to point out that a new literacy is emerging, other than traditional literacy (reading and writing) [5]. Programming was the key feature of this literacy. This metaphor can be taken further [6], in that the new literacy is based on the construction of a structure of dynamic symbols (a virtual world), embodying a dynamic, conceptual model of reality. This new literacy allows people to interact with simulations of reality in a dynamic way, adding a new dimension to reasoning over static symbolic models of this reality, as in traditional literacy.

Let us give an example. One of the projects Cetis has been working on is the so-called Mexx-case [7]. In this project, the working of the Mexx clothing company is simulated in the form of a virtual office with which a student can interact by visiting rooms and interviewing people. This allows the student to gain information that is needed to solve realistic problems confronting the Mexx Company. A multimedia application has been developed using Macromedia's Authorware. Also, a web site has been created that integrates the case into a LearningSpace [8] course. Thus far, interaction of students with the simulation is one-way only. Students gain information from the simulation, but their actions will not influence the simulation itself. Only when the student's actions influence the behaviour of the Mexx simulation will a "real" virtual world have been realised.

\subsection{How does higher education feel about innovation and ICT?}

The Dutch Ministry of Education, Culture and Science recently commissioned a study [9] into the use, opinions, and expectations of ICT in higher education in the Netherlands. All the faculties of all the Dutch universities were approached, achieving a response rate of $71 \%$, covering $76 \%$ of the student population in higher education in the Netherlands. Conclusions of this survey are:

- ICT is not an isolated technological development, but must be seen in the light of its contribution to new forms of education; 
- $40 \%$ of Dutch faculties have developed a policy on ICT and education; $48 \%$ are working to develop such a policy;

- ICT enhances the quality of education;

- Use of ICT is expected to increase in the coming years;

- Use of generic applications and e-mail is increasing, but is not yet common for all students and teachers;

- Online learning environments, including GroupWare and the World Wide Web, will develop strongly in the immediate future;

- Use of professional applications for specific study profiles will increase in the immediate future, but developments are lagging behind in language and art;

- Important reasons for introducing ICT are flexibility, individual learning routes, combination of learning and working, and preparation for Lifelong Learning;

- Institutional Human Resource Management is weak and lacks vision;

- There is only incidental cooperation among universities and between universities and commercial enterprises;

- ICT is an important catalyst of educational innovation;

- Use of ICT in part-time, continuing, and cooperative education is increasing very quickly.

\subsection{From old to new: re-engineering the educational organisation}

Higher education seems to recognise the need for educational innovations with ICT. But is it capable of changing itself into "the" new learning organisation? Can it develop into a knowledge network in which students participate in interaction with colleagues (peers), experts (both on content and on learning process), and virtual environments (simulation, what if) at home, at the work/study place or somewhere else (using mobile communication). Is it able to change into a student-centred organisation and will it satisfy student demands? Among these demands are:

- a just fit of learning (personalised learning — I want it to my taste and needs);

- just in time learning - I need it now;

- learning in the right place - I want it here, not there;

- learning at the right time - I want it now, not then;

- learning in a realistic setting - I want to see that it is relevant;

- with immediate effect - I want to apply my new knowledge today;

- with concrete effect - WISIWIHL, What I See Is What I Have Learned. 
Will higher education be able to find added value in a focus on the "process" of learning rather than in a focus on content? Will it be able to enhance interactivity and feedback?

Realising all this requires a profound change in educational organisation. However, the management of this change is hindered by characteristics of the traditional educational organisation [10], in which autonomous professionals operate on the basis of professional skills and standards. Management control is not very effective: the driving force is disciplinary content. The educational standard also prohibits self-control by students: the teacher teaches content; students have to adapt. The teacher tests on content; the students have to learn by heart. The organisation is flat, because the teacher is autonomous in the classroom. And teacher-oriented standards help to enforce the status quo. Management control will have to be empowered to break through this status quo: educational management (which is not the same as educational administration) has to be introduced. Quality standards that put the student in the centre have to be accepted. Teachers will have to work in a matrix organisation: teams of teachers with teacher middle management (team manager).

The risk that "the" learning organisation cannot be realised is very real, because:

- there is no culture of change; the prevailing culture is one of the status quo, not of participation;

- educational management is not an accepted part of this culture;

- results are defined as teacher results, not student results.

Realisation of an ICT-supported educational environment therefore requires both managing organisational change and solving technologyrelated problems. It is no use to introduce information technology while not changing the organisation of education. In the latter case, the technology will be used to "deep freeze" the old organisation in technical automation, thereby reinforcing non-feasible characteristics. Also people's acceptance of the electronic environment is an issue. This acceptance is, in general, better with students than with teachers, but, even so, small obstacles in ease of use (e.g., a relatively long start-up time for an application) can prohibit adoption.

\subsection{Functionality of a higher education electronic environment}

"The" higher education learning environment is a student-centred working environment with the following characteristics:

- it is a knowledge network (in which information, peers, and experts have their place); 
- it offers professional tools for getting, exploiting, and processing/applying information;

- it facilitates communication with colleagues and experts (in the knowledge network);

- it offers interaction with virtual environments (simulation, what if);

- it is flexible in content, time, and place;

- tools, communication, and interaction are integrated into one environment;

- the environment is accessible at home, at the work/study place, or on the road (mobile communication);

- the environment can be adapted to student and teacher needs.

The starting point is the primary process: learning by the student in a knowledge network. Supporting this primary process is a secondary process of quality control: students account for their actions and results against recognised criteria of quality, inviting assessment by peers and experts. The tertiary process is that of coaching by teacher and educational institution.

\subsubsection{What student actions should the environment support?}

The integrated higher education electronic environment should support the following student actions:

- navigating through the environment;

- planning of the learning process using meta-structures of syntax and semantics;

- planning and tracking of quality assurance of the learning process and learning results;

- performing of the actual learning/working process:

- interaction with knowledge bases;

- interaction with content experts: peers and professionals;

- interaction with process experts: teachers;

- building of and interaction with dynamic models (virtual worlds, simulations);

- production of reports and products;

- presentation and communication of results.

- monitoring of progress and of quality of the learning/working process;

- evaluation of results, both the work produced by the students and what this work shows regarding their learning. 


\subsubsection{What functionality is needed?}

To support student actions, the higher education electronic environment must offer both generic and customised tools. The generic toolbox must offer:

- navigator;

- organiser;

- information browser;

- information systems (filing systems, data base systems);

- e-mail/e-voice/e-video system;

- GroupWare (including sharing of documents);

- text processor;

- spreadsheet;

- presentation tool.

Among the customised tools are:

- educational information systems (administrative systems, library systems, WWW sites);

- drill and practice applications;

- professional, discipline-specific tools (e.g., programming tools, CAD/CAM tools);

- professional, discipline-specific simulation tools.

\subsection{Integrated electronic learning environments}

An electronic learning environment will seek to combine the generic and customised tools of the electronic educational environment into one integrated environment offering the functionality needed by students. But there are also other requirements to be met. Requirements include [11], [12]:

- Application requirements;

- Design requirements;

- Learning and teaching requirements;

- Access requirements;

- Management requirements;

- User requirements;

- Communication requirements;

- Maintenance requirements.

A full overview over these requirements is given in Appendix A.

\subsection{An example: Lotus LearningSpace}

One of the many electronic learning environments available today is Lotus LearningSpace. LearningSpace is an addition to the Lotus Notes 
environment, a GroupWare environment with extended features supporting database-structures and web-compatibility. As stated in [8], "Lotus Notes provides a way for users to organise, share, collaborate, and integrate information in a secure environment. With Notes, you can seamlessly integrate the two worlds..." These two worlds are the world as we know it and a "virtual world", in this case an electronic educational environment. Lotus LearningSpace provides a number of standard database templates in the Lotus Notes environment, five to be exact, with functionality supporting educational activities. These five templates are Schedule, MediaCenter, CourseRoom, Profiles, and Assessments. These templates can be used to support diverse student activities, such as:

- working in teams using dedicated course materials (Schedule, MediaCenter, and CourseRoom);

- secure logging of personal student results and information (CourseRoom and Profiles);

- integrated incorporation of personal student annotations (Schedule, MediaCenter, and CourseRoom).

\subsubsection{Schedule (the planning centre)}

Schedule guides students through tasks ordered in a specific sequence or structure. These tasks may include initiation of discussions and assignments, varying from the assignment to read a chapter of a book to an assignment to look for specific information on the World Wide Web or in the digital library. Schedule is an organiser for the course: what students should do in which week, unit or module. It also provides an archive of the course material. If students miss a unit, they can catch up by going through the Schedule.

\subsubsection{MediaCenter (information network)}

MediaCenter supplies a structure for organising all the information used in the course. To this information, key words and labels may be attached, such as: date, writer, document type, and source. An extra option is the invocation of Schedule functions; for example, a discussion or an assignment can be initiated after the student has accessed specific information in the MediaCenter. The storage of information in the MediaCenter is only the limited only by Internet protocols. Complete programs can be stored in the MediaCenter, downloadable for students. HTML, JavaScript-code and Java applets can be included in documents. Embedded Authorware applications and RealMedia files may also be included. 


\subsubsection{CourseRoom (interaction network)}

In the CourseRoom, all interaction between the participants (students, teachers, experts) takes place in a way which strongly resembles a threaded newsgroup discussion. Students and teachers may initiate discussions, participate in discussions, and also include attachments. These discussions may be open or private. In the CourseRoom, students may submit their individual or group assignments. The power of LearningSpace lies in its support for teamwork; teams may work on an assignment in private; only after results are finalised are they submitted to the teacher.

\subsubsection{Profiles (customising the environment and "who's who?")}

In Profiles, students and teachers may reveal their faces, interests, and backgrounds to members of this virtual electronic world. Profiles also offers the possibility of configuring the system environment. For example, the MediaCenter may specify that a program has to be run which is stored locally on CD-ROM. Profiles then provides the system with the information where to find this $\mathrm{CD}$.

\subsubsection{Assessments (the testing centre)}

The fifth database supports testing, initiated by the teacher, and, if so specified, automatic correction. Several types of questions, such as true/false and multiple choice, can be used in these tests. Random choice from (a part of) an item-bank is also available. Several types of assessment are possible:

- self-assessment which allows students privately to evaluate their level of knowledge at some point or other in the course;

- graded assessment;

- evaluative assessment.

\subsection{Does LearningSpace meet the requirements?}

The functionality of digital learning environments, such as LearningSpace, needs to be enhanced with standard software tools (e.g., Lotus Office Suite or Microsoft Office Suite) and customised software tools (educational information systems, specific professional tools) to create an electronic higher education learning environment. Given this situation, the digital learning environments proper can be assessed against the criteria given in Appendix A. Two recent assessments have been made in the Netherlands covering sixteen digital learning environments [11], [12]. A first global assessment on suitability for use in higher education yielded five 
environments for further, detailed assessment: WebCT, TopClass, Oracle Online Learning Application, Lotus LearningSpace, Virtual Campus/EuroCampus. Conclusions were [12] that:

- WebCT and TopClass are a good choice when used in addition to traditional education.

In this situation, LearningSpace is recommendéd when (team) communication is indicated; Oracle Learning Application is recommended when content reuse is indicated or large numbers of students have to be served.

- LearningSpace, Oracle Learning Application and Virtual Campus/EuroCampus are a good choice when used to replace part of traditional education.

In this situation LearningSpace is recommended for team-based learning; Oracle Learning Application is recommended when content reuse is indicated or large numbers of students have to be served; Virtual Campus/EuroCampus is recommended when infrastructure is minimal.

- Oracle Learning Application, LearningSpace and Virtual Campus/EuroCampus are a good choice when a higher education digital learning environment is envisaged to replace traditional education. Oracle Learning Application is recommended when complete place and time independence is required; LearningSpace is recommended when flexibility in didactic and coaching approaches is required; Virtual Campus/EuroCampus is recommended when the institution does not want to get involved in development, maintenance and distribution of courses.

Experience within the Hogeschool van Utrecht with LearningSpace is in accords with the above assessment. LearningSpace has proven to be a stable environment, highly appreciated by both students and teachers. Approximately 40 teachers who develop their own courses currently use it. These teachers use LearningSpace to broaden the view of students beyond books and black- or whiteboard. Students use new media and learn how to use the World Wide Web to their own advantage. Group work features are highly appreciated and firmly used within several courses. Using LearningSpace, teachers feel invited to apply new media. However, pioneer teachers also see their investment in hours as a drawback and spend a lot of spare time developing their courses.

Students experience LearningSpace as new and different, and, because of that, as a challenge to use. LearningSpace as a method of learning received a high appreciation in a limited evaluation among students in one of the courses.

Maintenance of the LearningSpace system is relatively easy, on the condition that an infrastructure and operational knowledge exist for Lotus 
Notes and for the Domino system. Building this operational know-how is, however, no trivial investment. It must also be stressed that from the start a substantial initial investment must be made in structuring the technical and organisational infrastructure.

\section{APPENDIX A: REQUIREMENTS FOR INTEGRATED LEARNING ENVIRONMENTS}

A learning environment will seek to combine the generic and customised tools of the electronic educational environment into one integrated environment. Requirements for such a learning environment include the following (this overview is based on requirements listed in [11] and [12], but requirements haven been reorganised and extended).

\section{Application requirements}

- individual learning;

- cooperative learning;

- project work;

- problem/task oriented learning;

- constructive learning;

- self-supported learning.

Design requirements

- ease of design;

- templates;

- authorisation facilities;

- ease of updating;

- integration of audio and video.

\section{Learning and teaching requirements}

- scheduling and tracking;

- instruction;

- tasks (individual and group);

- registered testing;

- submission of reports;

- presentation support;

- different types of assessment;

- filing;

- printing.

\section{Access requirements}

- access to external digital resources;

- access to internal digital resources;

- access to the World Wide Web;

- access to educational information systems; 
- access to teachers;

- access to students;

- access to experts.

\section{Management requirements}

- course organisation;

- group definition;

- definition of learning route;

- monitoring of learning route;

- inspection of test results.

\section{User requirements}

- ease of use;

- navigation support;

- planning support;

- monitoring support;

- adaptable, individual definition of resources;

- individual adaptation of learning route;

- individual definition of tests;

- environment is extendable to include my preferred resources, tools etc.;

- easy to learn, easy to use;

- online help.

\section{Communication requirements}

- document sharing;

- group scheduling;

- e-mail;

- video-conferencing;

- chat box;

- white board;

- bulletin board;

- news group/ personal information centre;

- World Wide Web.

\section{Maintenance requirements}

- Web-based communication interface;

- consistent data base structure;

- Windows NT and Unix compatible;

- secure operation;

- fault monitoring;

- allowing authorisation;

- extendable;

- easy to install and maintain. 


\section{REFERENCES}

[1] M. Hammer \& J. Champy, Re-engineering the corporation, Nicolas Brealy Publishing, London, 1993.

[2] T. J. van Weert, Informatics and the organization of education, in: B. Samways and T. J. van Weert (eds.), The impacts of informatics on the organization of education, Elsevier Science Publishers B. V., Amsterdam, 1992, p. 15-24.

[3] H. Berghel, Digital Village: Value added publishing, Communications of the ACM, Volume 42, Number 1, January 1999, p. 19-23.

[4] http://www.cave.vt.edu/ . "CAVE" is a trademark name of the Electronic Visualization Laboratory of the University of Illinois.

[5] P. Ershov, Programming; the second literacy, in: Proceedings of the Third World Conference on Computers in Education, R. Lewis \& D. Tagg, eds., North-Holland Publ. Co., Amsterdam, 1981, p. 1-7.

[6] T. J. van Weert, Literacy in the information age, in: B. Sendov and I. Stanchev, eds., Children in the information age, Pergamon Press, Oxford, 1988, p. 109-122.

[7] http://www.cetis.hvu.nl/cleo7/

[8] Working with Lotus Notes and the Internet, Lotus Development Corporation, Cambridge, MA 02142, 1996.

[9] Flexibel en open hoger onderwijs met ICT, Een inventarisatie van gebruik, meningen en verwachtingen (Flexible and open higher education with ICT, An inventarisation of use, opinions and expectations), in opdracht van het Ministerie van OC\&W (commissioned by the Dutch Ministry of Education, Culture and Science), IVLOS Universiteit Utrecht and OC/OT Universiteit Twente, Utrecht/Enschede, 1999 (in Dutch), p. 9-11, p. 51-59.

[10] H. Mintzberg, "The professional bureaucracy", in: J. B. Quinn, H. Mintzberg, R.M. James, The strategy process, Prentice Hall, Englewood Cliffs NY, 1988.

[11] J. Droste, Een vergelijkend onderzoek naar tele-leerplatforms (Digital learning environments: A comparative study), CINOP, Den Bosch, 1998 (in Dutch).

[12] Advies keuze teleleerplatform 1999 (How to choose a digital learning environment), SURF Educatie $<F>$, Utrecht, 1999 (in Dutch).

${ }^{i}$ Oracle has recently announced that Oracle Online Learning Application will be completely redesigned; development of the new system will take at least one and a half years. 